\title{
Charge Trapping in Mixed Organic Donor-Acceptor Semiconductor Thin Films
}

\author{
Shota Nunomura,* Xiaozhou Che, and Stephen R. Forrest**
}

\begin{abstract}
Charge trapping is an important factor that often limits the performance of optoelectronic devices. Specifically, accumulation of trapped charges in the active layer of organic photovoltaic (OPV) cells impacts charge transport by inducing band bending, and reduces the charge carrier lifetime and density by accelerating recombination. ${ }^{[1-5]}$ Among the various origins of trapped charges, ${ }^{[6-8]}$ those associated with film morphology are particularly important for small-molecule organic semiconductors. ${ }^{[8]}$ This class of materials can form self-organized nanostructures in neat films, ${ }^{[9-11]}$ and often domains or amorphous morphologies are found in blends of more than one molecule, as in the case of high performance mixed donor (D)-acceptor (A) heterojunctions. ${ }^{[12,13]}$ In such mixtures, charges can be trapped at grain boundaries ${ }^{[6]}$ or at the interfaces between D and A domains. ${ }^{[14]}$ Thus, characterization of trapped charges is important for understanding charge transport and improving device performance. Here we describe a simple pump-probe method whereby trapped charges are optically induced to contribute to the total photocurrent. Measurement of the incremental trap current thereby provides an accurate and quantitative determination of the trapped charge density in organic semiconductor thin films, and in particular donor-acceptor blends ${ }^{[2]}$ commonly used in high efficiency and high reliability OPVs. The technique is applied to neat small-molecule films as well as D-A mixtures comprised of compounds used in high performance OPV cells. The trapped charge density is correlated to the performance of cells made from these same materials, and the correlation is discussed in terms of the charge transport dependence on thin film morphology.

Charge trapping in the active layer of OPVs has previously been studied using impedance spectroscopy, ${ }^{[15]}$ deep-level
\end{abstract}

Dr. S. Nunomura, Prof. S. R. Forrest

Department of Electrical Engineering

and Computer Science

University of Michigan

Ann Arbor, Michigan 48109, USA

E-mail:s.nunomura@aist.go.jp; stevefor@umich.edu

Dr. S. Nunomura

Research Center for Photovoltaic Technologies

National Institute of Advanced Industrial Science and Technology

Tsukuba, Ibaraki 305-8568, Japan

X. Che, Prof. S. R. Forrest

Applied Physics Program

University of Michigan

Ann Arbor, Michigan 48109, USA

Prof. S. R. Forrest

Department of Materials Science and Engineering

University of Michigan

Ann Arbor, Michigan 48109, USA transient spectroscopy, ${ }^{[16,17]}$ transient photocurrent, ${ }^{[18]}$ and thermally stimulated current. ${ }^{[19,20]}$ It has been found that trapping is strongly dependent on the particular combination of D-A molecules and illumination conditions employed. However, the important problem of the dependence of charge trapping on the content and concentration of D-A mixtures and on film morphology has not yet been thoroughly investigated. Indeed, previous investigations have provided the density of gap states and/or trap site densities; ${ }^{[15-20]}$ but, to our knowledge, a quantitative determination of the relationship between OPV performance and the density of trapped charges has not been reported.

Using the experimental set up in Figure 1, we illuminate the sample under investigation by a relatively intense, high-energy optical pump that generates excitons that subsequently dissociate into free charges (i.e., polarons). These charges result in a photocurrent $\left(I_{\mathrm{p}}\right)$, while a small fraction populate trap states within the organic energy gap. The trapped charges are subsequently depopulated by a delayed, low-energy pulse that induces an incremental increase in photocurrent due to traps $\left(I_{\text {trap }}\right) \cdot{ }^{[21,22]}$ To quantitatively determine the free and trapped charge densities, their respective generation rates are given by:

$\mathrm{g}_{\mathrm{HOMO}}=n_{\text {Номо }} \sigma_{\text {Номо }} \Gamma_{\text {рump }}$

$g_{\text {trap }}=n_{\text {trap }} \sigma_{\text {trap }} \Gamma_{\text {probe }}$

where $n$ is the density of electrons in the highest occupied molecular orbital (HOMO) and in traps, denoted by the subscripts, HOMO and trap, respectively. Also, $\sigma$ is the photoabsorption cross-section, and $\Gamma_{\text {pump,probe }}$ are the single pass photon flux generation efficiency of the pump and probe, respectively. The relative absorption efficiency by traps vs at the HOMO is given by the ratio, $\sigma_{\text {HOMO }} / \sigma_{\text {trap }}$. The loss of polarons is dominated by trap mediated Shockley-Read-Hall recombination, where the loss rate is determined by the charge lifetime, $\tau_{\mathrm{r}}$. In steady state, the electron-polaron density in the lowest unoccupied MO (LUMO), $n_{\text {LUMO, }}$, is then:

$n_{\mathrm{LUMO}}=g_{\text {HOMO,trap }} \tau_{\mathrm{r}}$

Using Equation 1-3, the currents, $I_{\mathrm{p}}$ and $I_{\text {trap }}$, induced by pump and probe illumination are:

$I_{\mathrm{p}}=e \eta_{\mathrm{CC}} g_{\text {номо }} \tau_{\mathrm{r}} v_{\mathrm{d}}$

$I_{\text {trap }}=e \eta_{\mathrm{CC}} g_{\text {trap }} \tau_{\mathrm{r}} v_{\mathrm{d}}$ 


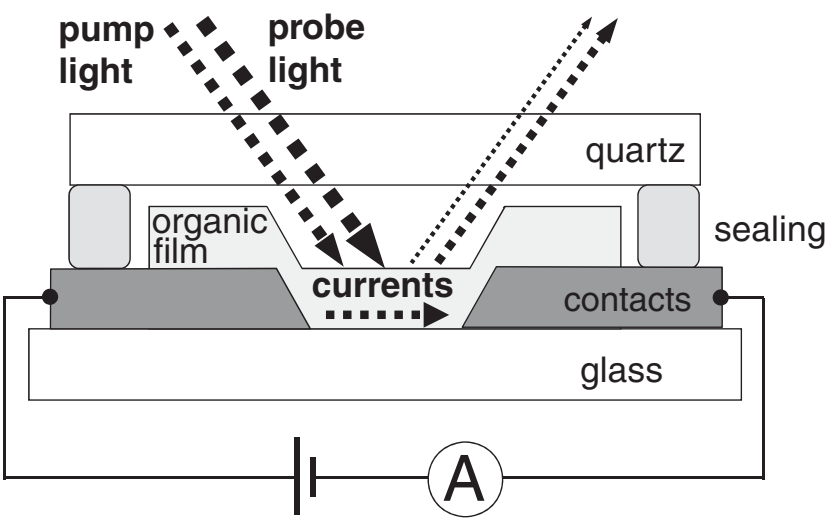

Figure 1. Schematic of the measurement and sample configurations. The organic semiconductor film is illuminated with both the short wavelength pump and long wavelength probe illumination. The pump induces the photocurrent in the film while the probe beam induces a smaller current via depopulation of traps. The currents are collected at the interdigitated contacts on the substrate. The film is packaged to prevent exposure to the environment.

Here, $e$ and $v_{\mathrm{d}}$ are the elementary charge and its drift velocity, respectively, and $\eta_{\mathrm{CC}}$ is the charge collection efficiency. Taking the ratio of $I_{\text {trap }}$ to $I_{\mathrm{p}}$, we obtain the trapped-to-HOMO charge density ratio:

$$
\frac{n_{\text {trap }}}{n_{\text {HОмO }}}=\left[\frac{\sigma_{\text {Hомо }}}{\sigma_{\text {trap }}}\right]\left[\frac{\Gamma_{\text {pump }} I_{\text {trap }}}{\Gamma_{\text {probe }} I_{\mathrm{p}}}\right]
$$

The surface morphology and nanostructure of the organic films are shown in Figure 2. The neat $\mathrm{C}_{60}$ film is characterized by grains separated by sharp boundaries (dark regions, Figure $2 \mathrm{a})$, resulting in a root mean square $(\mathrm{rms})$ roughness of $2.2 \pm 0.2 \mathrm{~nm}$. The grain sizes range from several to a few tens of nanometers. The film is a polycrystalline, face centered cubic structure, as indicated by SAED patterns in Figure $2 \mathrm{~b}$. The diffraction rings, corresponding to the (111) and (220) planes are labeled. In contrast, the D-A mixed films are comparatively smooth, with rms roughness $=0.5 \pm 0.05 \mathrm{~nm}$ at the donor concentration of $n_{\mathrm{D}}=50 \%$ (Figure 2c). Such surface smoothness is observed for $n_{\mathrm{D}}$ between $20 \%$ and $80 \%$. The SAED patterns for the mixtures in Figure $2 \mathrm{~d}$ are diffuse, indicating a more disordered, isotropic morphology.

Figure 3 shows the time evolution of $I_{\mathrm{p}}$ and $I_{\text {trap }}$ for a neat $\mathrm{C}_{60}$ film. With the exception of the brief parasitic transient due to
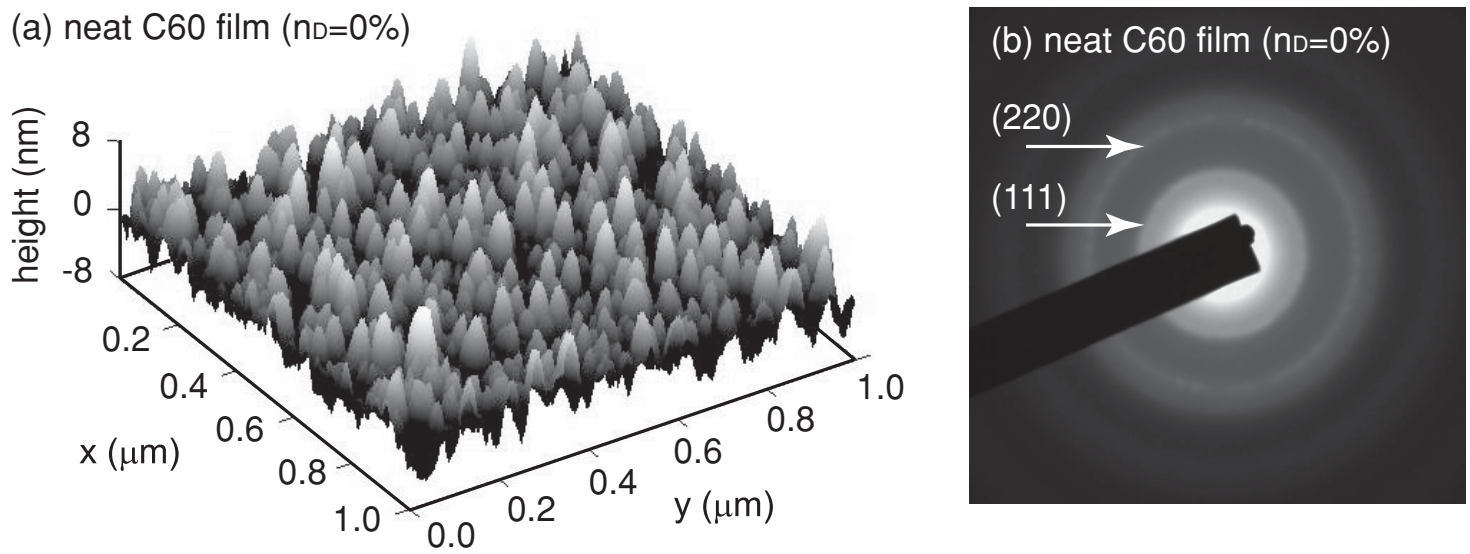

(c) D-A mixed film ( $\left.\mathrm{n}_{\mathrm{D}}=50 \%\right)$
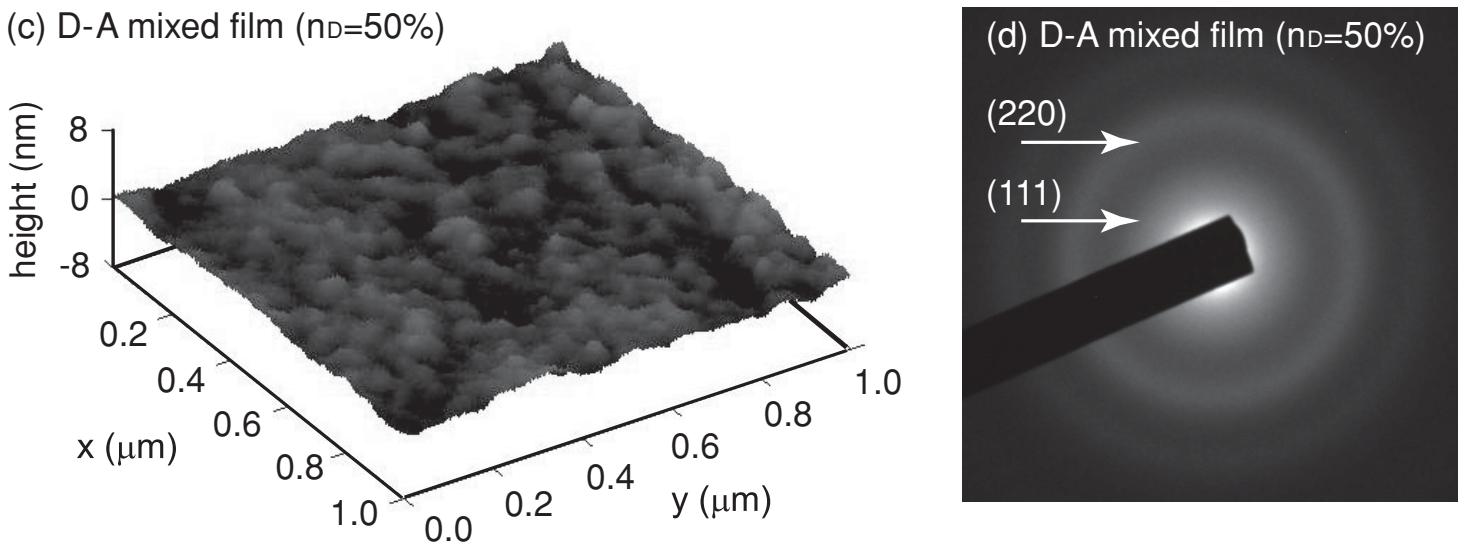

Figure 2. Film morphological analysis. a) Atomic force microscopy (AFM) image, and b) selected area electron diffraction (SAED) pattern of a neat $\mathrm{C}_{60}$ film. c) AFM image and d) SAED pattern of a 1:1 DTDCTB: $\mathrm{C}_{60}$ mixed film. The neat film is characterized by a granular morphology with a root mean square roughness of $r m s$ roughness $=2.2 \pm 0.2 \mathrm{~nm}$, whereas the mixed film surface is relatively smooth with $r m s$ roughness $=0.5 \pm 0.05 \mathrm{~nm}$. The nanostructure changes from polycrystalline (face-centered cubic structure) for the $C_{60}$ to less-ordered for the mixed films. 


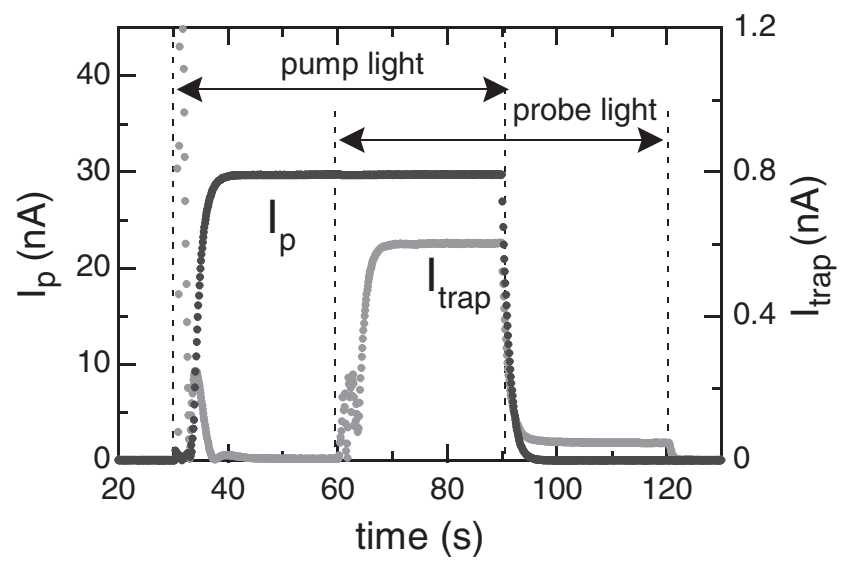

Figure 3. Time evolution of the $\mathrm{C}_{60}$ photo- and trap currents under pump and probe illumination. The trap current, $I_{\text {trap }}$, is significant only when photocurrent, $I_{\mathrm{p}}$, is generated under pump illumination during the time window from $60 \mathrm{~s}$ to $90 \mathrm{~s}$. The small $I_{\text {trap }}$ following $I_{\text {pump }}$ results from emission of residual trapped charge.

saturation of the lock-in amplifier at the onset of the pump, $I_{\text {trap }}$ is significant only when the pump is present, between $t=60 \mathrm{~s}$ and $90 \mathrm{~s}$. Once the pump illumination is terminated, $I_{\text {trap }} \rightarrow 0$, indicating that $I_{\text {trap }}$ is due only to carriers excited from trapping sites. An offset in $I_{\text {trap }}$ observed following the pump illumination ( $t=90-120 \mathrm{~s})$ is due to thermal emission of residual trapped charge that vanishes once the probe is turned off.

In Figure 4, we find that $I_{\text {trap }}$ saturates at high pump power $\left(P_{\text {pump }}\right)$, whereas $I_{\mathrm{p}}$ increases linearly with $P_{\text {pump }}$, i.e., $I_{\mathrm{p}} \propto P_{\text {pump }}$, suggesting an intensity-independent photogeneration efficiency. Correspondingly, $n_{\text {trap }} / n_{\text {HOMO }}$ determined from Equation 6 also saturates at high $P_{\text {pump }}$ due to nearly complete occupation of the trap sites. That is, at high $P_{\text {pump }}, n_{\text {trap }} / n_{\text {HOMO }} \approx 10^{-4}$.

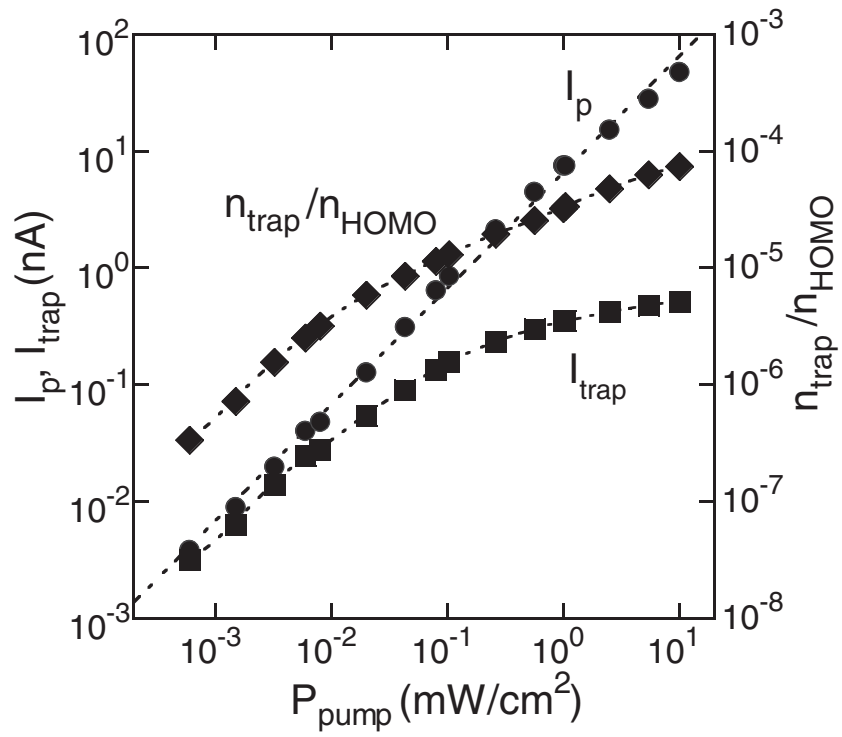

Figure 4. Pump (trap) current, $I_{\mathrm{p}}\left(I_{\text {trap }}\right)$ and trapped-to-highest occupied molecular orbital (HOMO) charge density, $n_{\text {trap }} / n_{\text {HOMO }}$, of a neat $C_{60}$ film as a function of pump power, $P_{\text {pump }}$. As $P_{\text {pump }}$ increases, $I_{\text {trap }}$ tends to saturate while $I_{\mathrm{p}}$ linearly increases, indicative of a constant quantum efficiency.
By assuming $\sigma_{\mathrm{HOMO}} / \sigma_{\text {trap }}=1$ (i.e., the donors, and the species or defects acting as traps have nearly equal cross sections) and $n_{\text {HOMO }}=10^{21} \mathrm{~cm}^{-3}$ corresponding to the approximate acceptor molecular density, then $n_{\mathrm{t}} \approx 10^{17} \mathrm{~cm}^{-3}$.

To ensure that the trap current provides a quantitative measurement of the density of trapped charges, we need to determine the significance of other possible current sources. These might include the current due to charge retrapping, the effects of free-carrier absorption, and the generation of charge due to low energy triplet and/or charge-transfer exciton dissociation. To estimate the importance of charge retrapping, in Figure 5a we plot $I_{\text {trap }}$ vs $P_{\text {probe }}$ and find that the trap current is linear over the entire range of probe powers tested. Since the experiments are performed in steady state, retrapping would be detected by a reduction from the linear trend as power is decreased, which is clearly not observed. In addition, free-carrier absorption can be discounted due to the low charge density (ca. $10^{12}-10^{13} \mathrm{~cm}^{-3}$ under AM1.5 1-sun illumination) compared to that of the measured trapped charge density $\left(10^{15}-10^{17} \mathrm{~cm}^{-3}\right.$, see below). Finally, the existence of low energy triplet or charge transfer excitons in the blends is not apparent from the near infrared absorption spectrum between the wavelengths of $800 \mathrm{~nm}$ and $2 \mu \mathrm{m}$ shown on a logarithmic scale in Figure $5 \mathrm{~b}$. Note that the sensitivity of the absorption data are sufficient to observe these effects, since $I_{\text {trap }}$ ranges from $1 \%$ to $10 \%$ of $I_{\mathrm{p}}$ (Figure $6 \mathrm{a}$ ). Moreover, the oscillator strengths of triplet and charge transfer state absorption are a very small fraction of singlets, thus allowing us to confidently rule out contributions to $I_{\text {trap }}$ from these potentially parasitic sources.

Figure 6a shows $I_{\mathrm{p}}, I_{\text {trap }}$, and $n_{\text {trap }} / n_{\text {HOMO }}$ for DTDCTB: $C_{60}$ mixed films as functions of the donor concentration, $n_{\mathrm{D}}$. Interestingly, $n_{\text {trap }} / n_{\text {HOMO }}$ is reduced by a factor of ten as $n_{\mathrm{D}}$ is increased from $n_{\mathrm{D}}=0 \%$ (neat $\mathrm{C}_{60}$ ) to $20 \%$ (A-rich mixed film). When $n_{\mathrm{D}}$ is increased to $50 \%, n_{\text {trap }} / n_{\text {HOMO }}$ reaches a minimum of $(3 \pm 1.5) \times 10^{-7}$, and thereafter, monotonically increases. Further, $I_{\mathrm{p}}$ increases with $n_{\mathrm{D}}$ due to efficient exciton dissociation in the mixtures, as expected. The maximum $I_{\mathrm{p}}$ is obtained at $n_{\mathrm{D}} \approx 20 \%$.

The performances of OPV cells are also influenced by the D-A mixture, as shown in Figure $6 \mathrm{~b}, \mathrm{c}$. The highest power conversion efficiency of $P C E=4.9 \pm 0.1 \%$ is achieved at $n_{\mathrm{D}} \approx 50 \%$. From Figure $6 \mathrm{~b}, \mathrm{c}$, the $P C E$ variation with donor concentration directly follows changes in the fill factor, FF. We find that FF decreases monotonically, from $0.54 \pm 0.01$ to $0.33 \pm 0.01$, as $n_{\text {trap }} / n_{\text {HOMO }}$ increases from $(3 \pm 1.5) \times 10^{-7}$ to $(5 \pm 2.5) \times 10^{-6}$, as shown in Figure 7.

As shown in Figure 2, the film morphology changes from granular to smooth for the neat and mixed films, respectively. Correspondingly, the crystalline order of the film is decreased as is the trapped charge density, from $n_{\text {trap }} / n_{\mathrm{HOMO}}=(2.5 \pm 1.5) \times 10^{-5}$ at $n_{\mathrm{D}}=0 \%$ to $(3.0 \pm 1.5) \times 10^{-7}$ at $n_{\mathrm{D}}=50 \%$. The high $n_{\text {trap }} / n_{\text {Номо }}$ in the neat $\mathrm{C}_{60}$ film suggests that charges are trapped at the grain boundaries, as illustrated in Figure 8a.

As shown in Figure $6 \mathrm{a}, n_{\text {trap }} / n_{\text {HOMO }}$ is U-shaped relative to $n_{\mathrm{D}}$, with a minimum at $n_{\mathrm{D}} \approx 50 \%$. This suggests that charge trapping occurs at the $\mathrm{D}-\mathrm{A}$ nanocrystalline domain boundaries $^{[6]}$ in the mixed films. For DTDCTB: $\mathrm{C}_{60}$ mixtures, the D-A interfaces form a transport barrier of $0.56 \mathrm{eV}$ for electrons and $0.9 \mathrm{eV}$ for holes as calculated from the LUMO and HOMO 
(a)

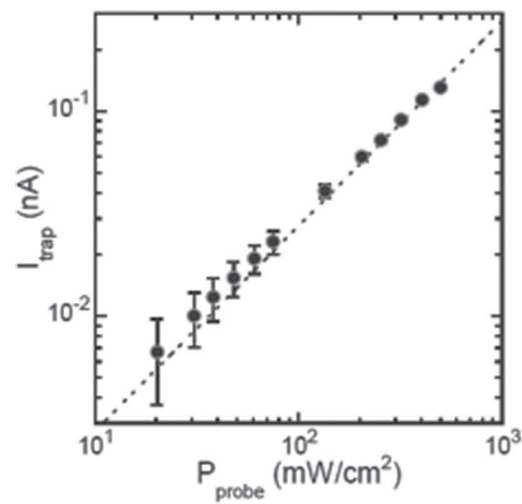

(b)

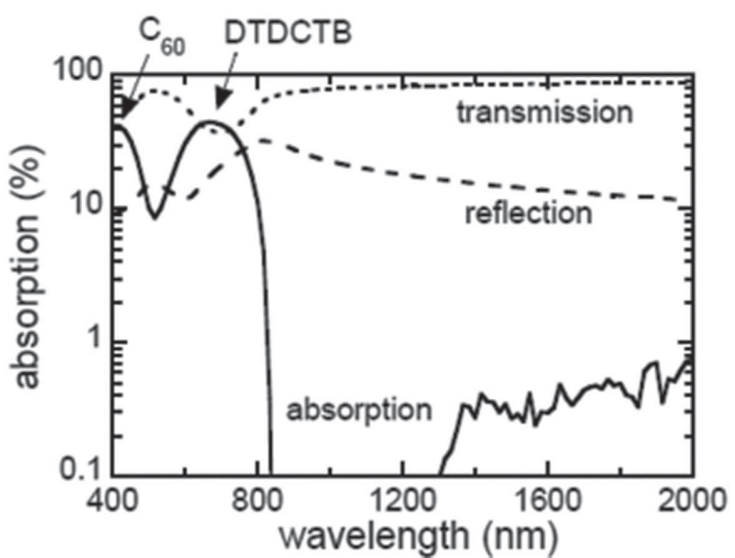

Figure 5. a) Trap current, $I_{\text {trap }}$, in a neat $C_{60}$ film as a function of probe power, $P_{\text {probe }}$. The trap current is linear over the entire range of probe powers studied. b) Absorption spectrum (solid line) of a 1:1 DTDCTB: $C_{60}$ mixed film at a thickness of $100 \mathrm{~nm}$. The absorption is obtained from transmission and reflection measurements also shown. The peak at $700 \mathrm{~nm}$ is due to absorption by DTDCTB. The mixed film is highly transparent over the near infrared region (wavelengths $>800 \mathrm{~nm}$ ). The apparent gap in absorption between 800 and $1300 \mathrm{~nm}$ is due to data lying below the detection noise floor at $\leq 0.1 \%$.

energy offsets between these molecules. Hence, electron transport across $\mathrm{C}_{60}$ domains is blocked by donor domains at high $n_{\mathrm{D}}$, whereas the transport of holes is blocked by acceptor molecules at low $n_{\mathrm{D}}$, as illustrated in Figure 8b,c. These boundaries

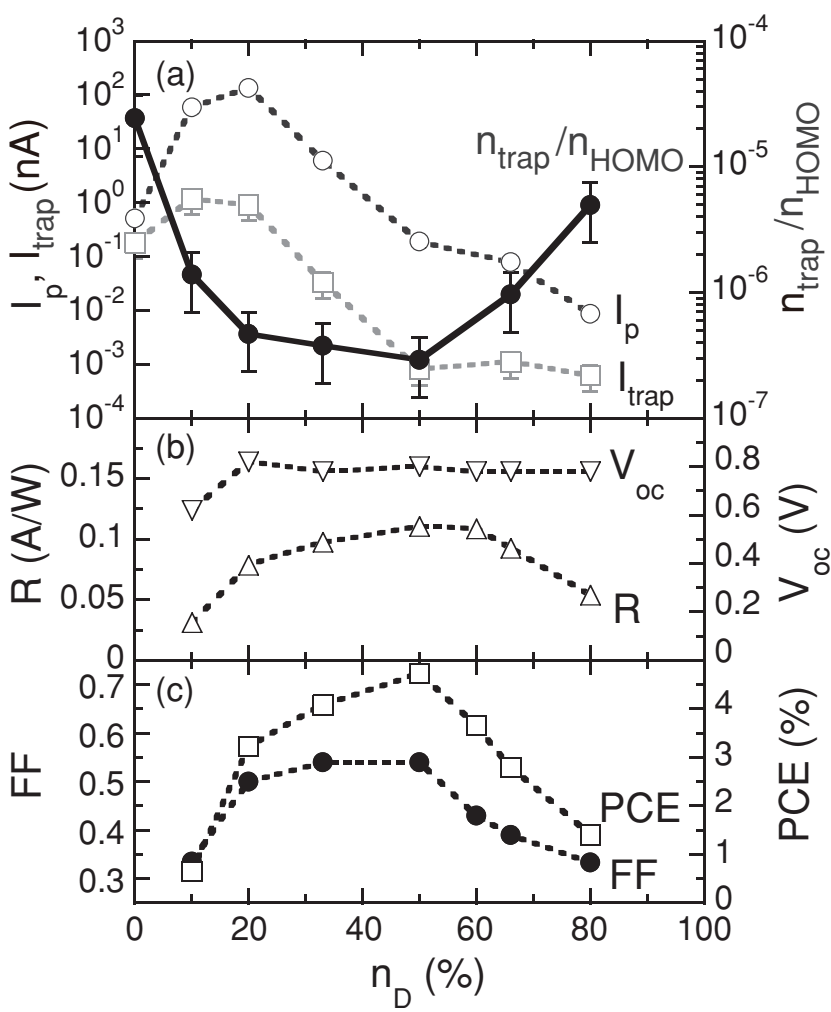

Figure 6. Organic photovoltaic cell performance characteristics, and $n_{\text {trap }} / n_{\text {HOMO }}$ as functions of donor concentration $n_{\mathrm{D}}$. a) $I_{\mathrm{p}}, I_{\text {trap }}$ and $n_{\text {trap }} / n_{\text {HOMO }}$, b) responsivity, $R$ (i.e., photocurrent per pump power) and open circuit voltage, $V_{o c}$, c) fill factor, $F F$, and power conversion efficiency, $P C E$. The PCE variation with $n_{D}$ directly follows changes in $F F$. The photo- and trap currents were measured under $P_{\text {pump }}=0.1 \mathrm{~mW}$ and $P_{\text {probe }}=500 \mathrm{~mW}$, respectively. are minimized (along with $n_{\text {trap }} / n_{\text {HOMO }}$ ) for approximately equal concentrations of donor and acceptor molecules, at which point continuous percolating pathways are formed that transport holes and electrons, respectively. We further note that grain boundary trapping depends on the energy offsets characteristic of a particular D-A composition, which can also be studied using this pump-probe technique.

Charge trapping by this same mechanism is observed in D-A mixed film OPV cells where we find in Figure 7 that FF is a strongly decreasing function of $n_{\text {trap }} / n_{\text {HOMO }}$. Indeed, when $n_{\text {trap }} / n_{\text {HOMO }}=(3 \pm 2) \times 10^{-6} \mathrm{~cm}^{-3}, F F$ is only $34 \pm 3 \% \mathrm{com}-$ pared to $F F=55 \pm 4 \%$ at $n_{\text {trap }} / n_{\text {HOMO }}=(3 \pm 2) \times 10^{-7}$. Hence, our work provides direct evidence that $\mathrm{D}-\mathrm{A}$ interface recombination leads to the commonly observed dependence of $F F$ in mixed heterojunction OPV devices on the mixture ratio. ${ }^{[4,23-25]}$

Finally, it is interesting to note that the electron-polaron trap density at grain boundaries in $\mathrm{C}_{60}$ films is roughly equal to the saturation exciton trap density (also ca. $10^{17} \mathrm{~cm}^{-3}$ ) found after aging $\mathrm{C}_{60}$-based planar OPVS ${ }^{[26]}$ This might indicate that grain boundaries are particularly vulnerable sites for the formation

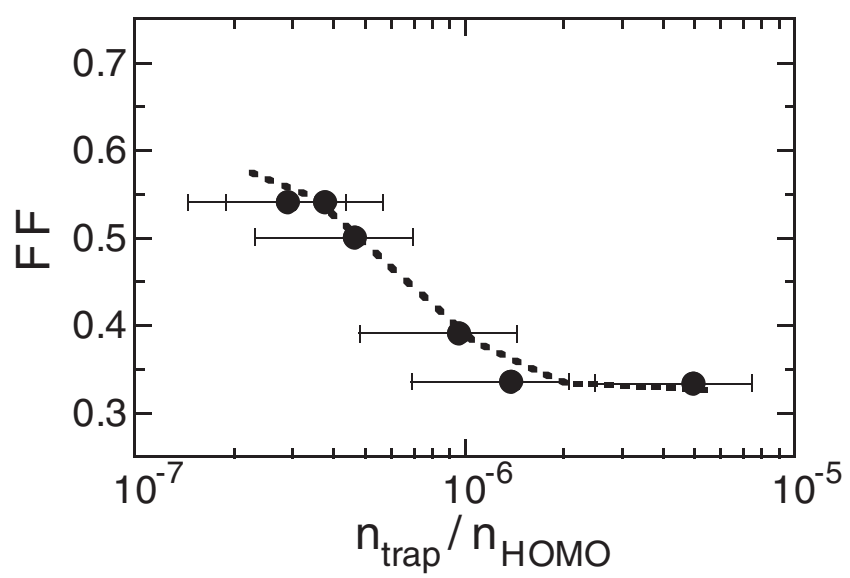

Figure 7. Fill factor, $F F$, vs $n_{\text {trap }} / n_{\text {HOMO }}$. As $n_{\text {trap }} / n_{\text {HOMO }}$ increases, $F F$ monotonically decreases. The dotted line is a guide to the eye. 
(a) neat C60 film

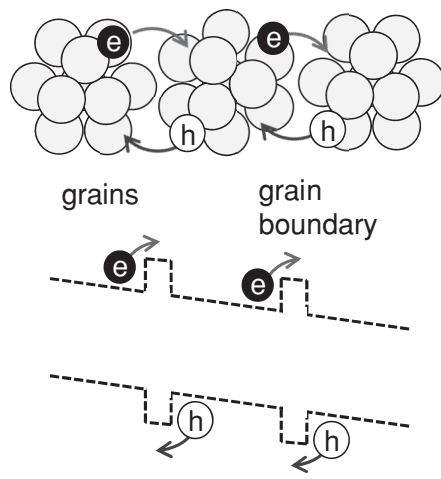

(b) A-rich mixed film

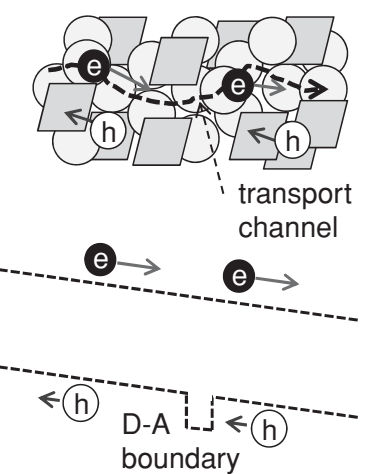

(c) D-rich mixed film

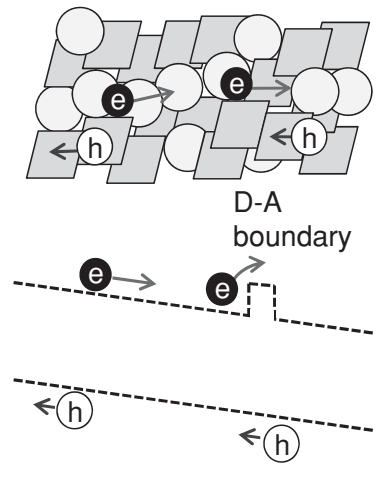

Figure 8. Charge carrier transport (upper figures) and energy level diagrams (lower figures) of the materials systems studied. a) Neat, granular $\mathrm{C}_{60}$, b) uniform smooth acceptor (A)-rich mixed films, c) uniform smooth donor (D)-rich mixed films. Charge carriers are trapped at grain boundaries in (a), whereas they are trapped at D-A interfaces in the mixed films. The grain boundaries and D-A interfaces behave as barriers to charge transport.

of exciton recombination centers due to preferential diffusive ingress of contaminants as compared to that in the film bulk. While more work is required to test the validity of this or other mechanisms, the pump-probe method provides a powerful tool for investigating the existence, evolution and effects of defect states.

We have demonstrated an optical pump-probe technique for determining the density of trapped charges in small-molecule organic semiconductor films. The trapped charge density was determined from the photo- and trap-induced currents using a model based on the charge generation-loss kinetics. It was found that in DTDCTB: $\mathrm{C}_{60}$ mixed films, the trap charge density depends on the ratio of donor to acceptor molecules. The donor concentration, $n_{\mathrm{D}}$, is found to influence $F F$ in mixed heterojunction OPV cells, with energy offsets between donor and acceptor domains resulting in charge trapping between $\mathrm{D}$ and A nanocrystalline grain boundaries. We find that an inherent limitation to mixed heterojunction device performance can be mitigated by the appropriate choice of $\mathrm{D}-\mathrm{A}$ pairs that minimize energy offsets while not adversely affecting the exciton dissociation efficiency. Finally, our results suggest the pump-probe method is a powerful means for investigating charge populations and their origins in a wide range of organic semiconductor thin films.

\section{Experimental Section}

The trapped charges were detected in organic films deposited on quartz substrates with interdigitated contacts forming $200 \mu \mathrm{m}$ long by $5 \mathrm{~mm}$ wide channels, shown in Figure 1 along with the optical set-up. The contacts themselves consisted of a $200 \mathrm{~nm}$ thick Ag layer covered with a $40 \mathrm{~nm}$ thick Ga-doped $\mathrm{ZnO}$ electron transport layer deposited via radio frequency magnetron sputtering through a shadow mask. The in-plane sample geometry is convenient for coupling optical illumination by multiple beams at different wavelengths.

We studied the donor, 2-\{[7-(5-N,N-ditolylaminothiophen-2-yl)-2,1,3benzothiadiazol-4-yl]methylene\}malononitrile $e^{[13,27,28]}$ (DTDCTB), and the acceptor, $\mathrm{C}_{60}$. The DTDCTB and $\mathrm{C}_{60}$ molecules absorb at wavelengths of $\lambda=500-800 \mathrm{~nm}$ and $350-650 \mathrm{~nm}$. The LUMO and HOMO energies of DTDCTB molecules are $3.44 \mathrm{eV}$ and $5.3 \mathrm{eV}$, respectively. ${ }^{[28]}$ For $\mathrm{C}_{60}$ molecules, the LUMO and HOMO energies are $4.0 \pm 0.1 \mathrm{eV}$ and $6.2 \pm 0.1 \mathrm{eV}$, respectively. ${ }^{[29]}$
The source materials were twice purified via thermal gradient sublimation to minimize the impurity concentration. The $D$ and $A$ molecules were then co-deposited on the substrate by vacuum thermal evaporation (VTE) in a system with base pressure ca. $10^{-7}$ Torr, whereby their D-A mixture ratio (with donor concentration, $n_{\mathrm{D}}$ ) was controlled by the individual molecular fluxes using quartz crystal monitors positioned over each source. Deposition at this low base pressure is also important in reducing impurity incorporation into the films. The $100 \mathrm{~nm}$-thick films were deposited at a rate of $0.1 \mathrm{~nm} / \mathrm{s}$ on the substrate at room temperature. The devices were subsequently packaged using a quartz lid sealed with ultraviolet cured epoxy to prevent environmental contamination during testing.

The surface morphologies of the films were determined with atomic force microscopy (AFM) over an area of $(1 \mu \mathrm{m})^{2}$. Selected area electron diffraction (SAED) patterns were used to characterize the nanocrystalline structure of the films.

The organic film was illuminated using pump light that generated excitons leading to the primary photocurrent, whereas the probe light excited trapped charge into the conduction level (see Figure 1). The photon energies of the pump and probe must be greater and less than the HOMO - LUMO gap energy, respectively. For this purpose, we used lasers emitting at wavelengths of $\lambda_{\text {pump }}=532 \mathrm{~nm}(2.33 \mathrm{eV})$ and $\lambda_{\text {probe }}=$ $1550 \mathrm{~nm}(0.8 \mathrm{eV})$. The laser powers were $P_{\text {pump }}=10 \mathrm{~mW}$ and $P_{\text {probe }}=$ $500 \mathrm{~mW}$, illuminating a $1 \mathrm{~cm}^{2}$ sample area at an incident angle of $45^{\circ}$.

The photocurrent and trap-induced current were collected by the interdigitated contacts biased at $10 \mathrm{~V}$. The pump and probe beam intensities were modulated at frequencies of $400 \mathrm{~Hz}$ and $290 \mathrm{~Hz}$, allowing the separate detection of the currents using lock-in amplifiers with integration times of $1 \mathrm{~s}$. The modulation frequencies were chosen to be sufficiently low for trapped charges to respond, while high enough to prevent heating by the high intensity long wavelength probe illumination.

The VTE-grown, $0.77 \pm 0.015 \mathrm{~mm}^{2}$ OPV cells used to study the correlation between the density of trapped charges and device performance have the following structure: glass/indium tin oxide (ITO) $(100 \mathrm{~nm}) / \mathrm{MoO}_{3}(10 \mathrm{~nm}) / \mathrm{DTDCTB} \mathrm{C}_{60}(40 \mathrm{~nm}) / \mathrm{C}_{60}(10 \mathrm{~nm}) /$ Bphen $(8 \mathrm{~nm}) / \mathrm{Ag}(100 \mathrm{~nm})$, where the D-A ratio was varied from device to device. We note that comparing data from the in-plane pump probe experiments with those of the vertical OPV structure is justified in the case of the blended films due to their isotropic structure implied from the SAED patterns in Figure 2. The device performances were evaluated in a glove box filled with ultrapure $(<0.1 \mathrm{ppm}) \mathrm{N}_{2}$ gas. The current density-voltage characteristics were measured under air mass (AM) 1.5G, 1-sun intensity illumination from a filtered Xe lamp. The intensity was calibrated using a National Renewable Energy Laboratory (NREL) traceable Si reference cell. The current and efficiency were corrected for spectral mismatch. ${ }^{[30]}$ 


\section{Acknowledgements}

The authors are grateful to J. D. Zimmerman, X. Tong, and N. Wang for their valuable comments. This work was supported by JSPS KAKENHI Grant Number 24540546 (SN, experiment, data analysis, theory) and by the US Department of Energy, Office of Basic Energy Sciences as part of Energy Frontier Research Centers: The Center for Solar and Thermal Energy Conversion at the University of Michigan (award DE-SC000957, XC, microanalysis, SRF, analysis, models).

Received: July 16, 2014

Revised: September 3, 2014

Published online: October 22, 2014

[1] V. D. Mihailetchi, J. Wildeman, P. W. M. Blom, Phys. Rev. Lett. 2005, 94, 126602.

[2] T. Kirchartz, B. E. Pieters, J. Kirkpatrick, U. Rau, J. Nelson, Phys. Rev. B 2011, 83, 115209.

[3] M. Kuik, L. J. A. Koster, G. A. H. Wetzelaer, P. W. M. Blom, Phys. Rev. Lett. 2011, 107, 256805.

[4] R. A. Street, A. Krakaris, S. R. Cowan, Adv. Funct. Mater. 2012, 22, 4608.

[5] G. G. Belmonte, Solid-State Electron. 2013, 79, 201.

[6] L. G. Kaake, P. F. Barbara, X.-Y. Zhu, J. Phys. Chem. Lett. 2010, 1, 628.

[7] L. Tsetseris, S. T. Pantelides, Phys. Rev. B 2011, 84, 195202.

[8] D. M. Pai, J. F. Yanus, M. Stolka, J. Phys. Chem. 1984, 88, 4714.

[9] R. Könenkamp, G. Priebe, B. Pietzak, Phys. Rev. B 1999, 60, 11804.

[10] Y. Yamamoto, H. Ichikawa, K. Ueno, A. Koma, K. Saiki, T. Shimada, Phys. Rev. B 2004, 70, 155415.

[11] J. D. Zimmerman, X. Xiao, C. K. Renshaw, S. Wang, V. V. Diev, M. E. Thompson, S. R. Forrest, Nano Lett. 2012, 12, 4366.

[12] S. Uchida, J. Xue, B. P. Rand, S. R. Forrest, Appl. Phys. Lett 2004, 84, 4218.
[13] S. W. Chiu, L. Y. Lin, H. W. Lin, Y. H. Chen, Z. Y. Huang, Y. T. Lin, F. Lin, Y. H. Liu, K. T. Wong, Chem. Commun. 2012, 48, 1857.

[14] L. J. A. Koster, V. D. Mihailetchi, P. W. M. Blom, Appl. Phys. Lett. 2006, 88, 052104.

[15] L. Burtone, J. Fischer, K. Leo, M. Riede, Phys. Rev. B 2013, 87, 045432.

[16] Y. S. Yang, S. H. Kim, J.-I. Lee, H. Y. Chu, L. M. Do, H. Lee, J. Oh, T. Zyung, M. K. Ryu, M. S. Jang, Appl. Phys. Lett. 2002, 80, 1595.

[17] A. Campbell, D. Bradley, E. Werner, W. Brutting, Synth. Met. 2000, 11, 273.

[18] Z. Li, C. R. McNeill, J. Appl. Phys. 2011, 109, 074513.

[19] J. Schafferhans, C. Deibel, V. Dyakonov, Adv. Energy Mater. 2011, 1, 655.

[20] R. Schmechel, H. Seggern, Phys. Status Solidi 2004, A201, 1215.

[21] S. Nunomura, I. Sakata, M. Kondo, Appl. Phys. Express 2013, 6, 126201.

[22] S. Nunomura, I. Sakata, AIP Adv. 2014, 4, 097110.

[23] B. P. Rand, J. Xue, S. Uchida, S. R. Forrest, J. Appl. Phys. 2005, 98, 124902.

[24] J. Xue, B. P. Rand, S. Uchida, S. R. Forrest, J. Appl. Phys. 2005, 98, 124903.

[25] H. Hoppe, M. Niggemann, C. Winder, J. Kraut, R. Hiesgen, A. Hinsch, D. Meissner, N. S. Sariciftci, Adv. Funct. Mater. 2004, 14, 1005.

[26] X. Tong, N. Wang, M. Slootsky, J. Yu, S. R. Forrest, Sol. Energy Mater. Sol. Cells 2013, 118, 116.

[27] L. Y. Lin, Y. H. Chen, Z. Y. Huang, H. W. Lin, S. H. Chou, F. Lin, C. W. Chen, Y. H. Liu, K. T. Wong, J. Am. Chem. Soc. 2011, 133, 15822 .

[28] H. W. Lin, H. W. Kang, Z. Y. Huang, C. W. Chen, Y. H. Chen, L. Y. Lin, F. Lin, K. T. Wong, Org. Electron. 2012, 13, 1925.

[29] Y. S. Gordeev, V. M. Mikushkin, V. V. Shnitov, Phys. Solid State 2000, $42,381$.

[30] C. H. Seaman, Sol. Energy 1982, 29, 291. 